\title{
Sodium butyrate activates NRF2 to ameliorate diabetic nephropathy possibly via inhibition of HDAC
}

\author{
Wenpeng Dong1,2, Ye Jia3 ${ }^{3}$ Xiuxia Liu4, Huan Zhang ${ }^{5}$, Tie Li6, Wenlin Huang7, \\ Xudong Chen ${ }^{8}$, Fuchun Wang ${ }^{6}$, Weixia Sun ${ }^{3}$ and Hao $\mathbf{W u}^{2,6}$ \\ 1Dialysis Center, Daqing Oilfield General Hospital, Daqing, Heilongjiang, People's Republic of China \\ 2Department of Nephrology, The Second Hospital of Jilin University, Changchun, Jilin, People's Republic of China \\ 3Department of Nephrology, The First Hospital of Jilin University, Changchun, Jilin, People's Republic of China \\ ${ }^{4}$ Department of Clinical Laboratory, The Second Hospital of Jilin University, Changchun, Jilin, People's Republic of China \\ ${ }^{5}$ Operating theatre, China-Japan Union Hospital of Jilin University, Changchun, Jilin, People's Republic of China \\ ${ }^{6}$ Department of Acupuncture and Tuina, Changchun University of Chinese Medicine, Changchun, Jilin, \\ People's Republic of China \\ 7School of Science and Technology, Georgia Gwinnett College, Lawrenceville, Georgia, USA \\ ${ }^{8}$ Gastroenterology Department No. 1, Jilin Central General Hospital, Jilin, Jilin, People's Republic of China
}

Correspondence should be addressed to W Sun or $\mathrm{H} \mathrm{Wu}$ Email sunweixia_78@163.com or wuhaobaha@yahoo.com, wuhaobaha@jlu.edu.cn

\begin{abstract}
Oxidative stress contributes to the pathogenesis of diabetic nephropathy (DN). Nuclear factor erythroid 2-related factor 2 (NRF2) plays a key role in cellular defense against oxidative stress. NRF2 activators have shown promising preventive effects on DN. Sodium butyrate $(\mathrm{NaB})$ is a known activator of NRF2. However, it is unknown whether NRF2 is required for $\mathrm{NaB}$ protection against $\mathrm{DN}$. Therefore, streptozotocin-induced diabetic C57BL/6 Nrf2 knockout and their wild-type mice were treated in the presence or absence of $\mathrm{NaB}$ for 20 weeks. Diabetic mice, but not NaB-treated diabetic mice, developed significant renal oxidative damage, inflammation, apoptosis, fibrosis, pathological changes and albuminuria. NaB inhibited histone deacetylase (HDAC) activity and elevated the expression of Nrf2 and its downstream targets heme oxygenase 1 and $\mathrm{NAD}(\mathrm{P}) \mathrm{H}$ dehydrogenase quinone 1. Notably, deletion of the $\mathrm{Nrf2}$ gene completely abolished $\mathrm{NaB}$ activation of NRF2 signaling and protection against diabetes-induced renal injury. Interestingly, the expression of Kelch-like $\mathrm{ECH}$-associated protein 1, the negative regulator of NRF2, was not altered by $\mathrm{NaB}$ under both diabetic and nondiabetic conditions. Moreover, NRF2 nuclear translocation was not promoted by NaB. Therefore, the present study indicates, for the first time, that NRF2 plays a key role in $\mathrm{NaB}$ protection against $\mathrm{DN}$. Other findings suggest that $\mathrm{NaB}$ may activate $\mathrm{Nrf} 2$ at the transcriptional level, possibly by the inhibition of HDAC activity.
\end{abstract}

Key Words

- diabetes

- KEAP1

- $\mathrm{NaB}$

- oxidative stress

- HDAC
Journal of Endocrinology (2017) 232, 71-83

\section{Introduction}

As one of the long-term complications of diabetes, diabetic nephropathy (DN) is the main cause of end-stage renal disease (Dronavalli et al. 2008). Thus, end-stage renal disease cannot be effectively prevented or treated. Therefore, there is an urgent need to develop effective methods to prevent or slow down the progression of DN. 
Oxidative stress contributes to the pathogenesis and development of DN (Zhang et al. 2012, Keshari et al. 2014). Nuclear factor erythroid 2-related factor 2 (NRF2) has been identified as a governor of antioxidant and redox signaling. NRF2 activates the transcription of downstream antioxidant genes such as heme oxygenase-1 (Ho1) and NAD(P)H dehydrogenase quinone 1 (Nqo1) (Ruiz et al. 2013). The elevated antioxidants function as cellular scavengers for free radicals, thereby preventing the cells from oxidative damage. We and others have demonstrated the beneficial effects of NRF2 activation on the prevention of DN in mice (de Haan 2011, Zheng et al. 2011, Wu et al. 2015a). Administration of the NRF2 activator sulforaphane (SFN) prevented diabetic renal damage, whereas the deletion of the Nrf2 gene completely abolished this action of SFN (Zheng et al. 2011, Wu et al. 2015a). These studies demonstrate that NRF2 is the key factor through which SFN ameliorates DN.

Sodium butyrate $(\mathrm{NaB})$ is known as an activator of NRF2 (Yaku et al. 2012, 2013, Liu et al. 2015). NaB significantly increases the expression of $\mathrm{Nrf2}$ and its downstream antioxidant genes, glutathione S-transferase and Nqo1, in interstitial epithelial cells in a dose-dependent manner (Yaku et al. 2012, 2013). Moreover, a recent study by Khan and coworkers showed that $\mathrm{NaB}$ ameliorated oxidative damage and fibrosis in the kidneys of diabetic rats along with a decrease in histone deacetylase (HDAC) activity (Khan \& Jena 2014). However, the mechanism by which $\mathrm{NaB}$ attenuates oxidative damage in $\mathrm{DN}$ or how much NRF2 contributes to this process remain unclear. Given that HDAC inhibition may facilitate the transcription of multiple genes, it is important to investigate which gene may play a key role in the protection of $\mathrm{NaB}$ against $\mathrm{DN}$.

Kelch-like ECH-associated protein 1 (KEAP1) sequesters NRF2 in the cytoplasm, restricting NRF2 from nuclear translocation and promoting its proteasomal degradation (Miyata et al. 2013). Several NRF2 activators such as SFN, curcumin and its analog, C66, enhance NRF2 function through the regulation of KEAP1 (Zhang \& Hannink 2003, Hu et al. 2011, Esatbeyoglu et al. 2012, Wu et al. 2016). Thus, it is interesting to test the effect of $\mathrm{NaB}$ on the expression of Keap1, which might be a mechanism by which NaB enhances NRF2 function, in addition to the HDAC-inhibiting activity of $\mathrm{NaB}$.

Therefore, this study aims to answer the following questions: (1) Is NRF2 required for $\mathrm{NaB}$ protection against DN in mice? (2) If required, how much does NRF2 contribute to the effect of NaB? (3) Is KEAP1 involved in $\mathrm{NaB}$ activation of NRF2 function in DN?

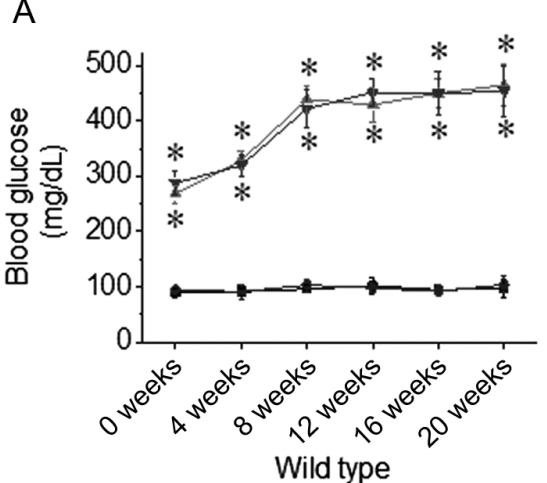

C

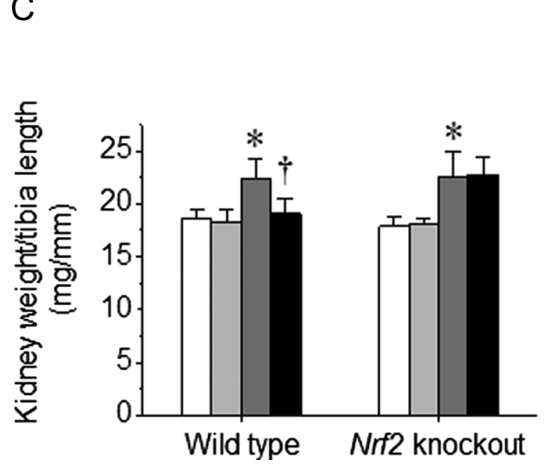

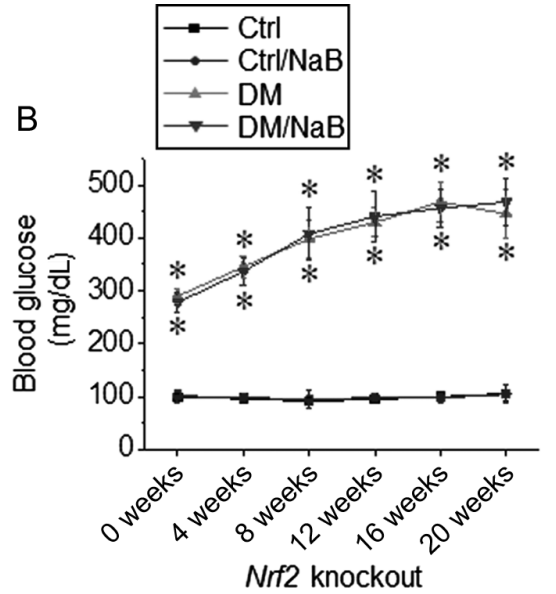

D

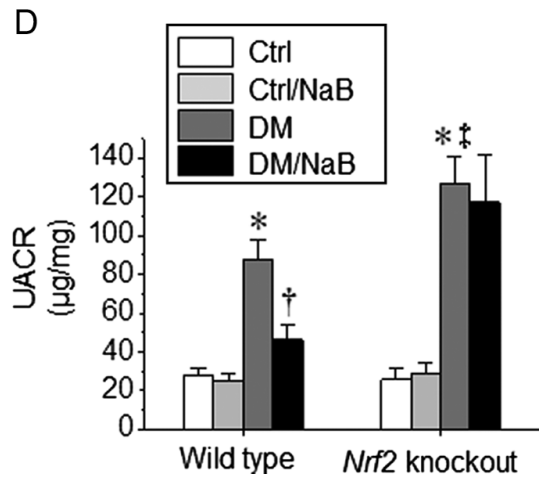

Figure 1

Deletion of the Nrf2 gene completely abolished $\mathrm{NaB}$ protection against diabetes-induced renal dysfunction. 8-week-old male C57BL/6 wild-type and $\mathrm{Nrf2-knockout} \mathrm{mice} \mathrm{were} \mathrm{induced} \mathrm{to}$ diabetes by streptozotocin. Blood glucose levels in (A) wild-type mice and (B) Nrf2-knockout mice were monitored at $0,4,8,12,16$ and 20 weeks after the onset of diabetes. Kidney weight, tibia length, urinary albumin and creatinine levels were determined with the ratios of (C) kidney weight to tibia length and (D) urinary albumin to creatinine (UACR) was calculated. Data are presented as means \pm S.D. $(n=7) . * P<0.05$ vs $C$ trl; ${ }^{\dagger} P<0.05$ vs DM; ${ }^{\ddagger} P<0.05$ vs wild-type DM. Lines: black, control (Ctrl); red, control treated with sodium butyrate (Ctrl/NaB); blue, diabetes (DM); green, diabetes treated with $\mathrm{NaB}(\mathrm{DM} / \mathrm{NaB})$. Bars: white, Ctrl; light grey, Ctrl/NaB; dark grey, $\mathrm{DM}$; black, DM/NaB. 
Accordingly, C57BL/6 male Nrf2-knockout mice and their wild-type controls were induced to diabetes through injection of streptozotocin (STZ). The mice were then treated in the presence or absence of $\mathrm{NaB}$ for 20 weeks.

\section{Materials and methods}

\section{Animal treatment}

C57BL/6 wild-type (Nrf2+/+) and Nrf2-knockout (Nrf2-/-) mice were obtained by the breeding of heterozygotes (Nrf2+/-) (Zheng et al. 2011). All mice were housed in the Animal Center of Changchun University of Chinese Medicine at $22^{\circ} \mathrm{C}$, on a $12: 12$-h light-darkness cycle with free access to rodent feed and tap water. The Institutional Animal Care and Use Committee at Changchun University of Chinese Medicine approved all experimental procedures for these animals. Consequently, this procedure was in accordance with the Guide for the Care and Use of Laboratory Animals by the US National Institutes of Health (2011, eighth edition).
Eight-week-old male mice received either sodium citrate or STZ (Sigma-Aldrich; $50 \mathrm{mg} / \mathrm{kg}$ daily, dissolved in $0.1 \mathrm{M}$ sodium citrate, $\mathrm{pH} 4.5$ ) through intraperitoneal injection for 5 consecutive days. One week after the last injection of STZ, fasting glucose levels (4-h fast) were measured. Mice with a fasting glucose level above $250 \mathrm{mg} / \mathrm{dL}$ were considered diabetic.

Diabetic and age-matched control mice were then given either a $\mathrm{NaB}$ diet or standard diet, as described in a previous publication (Gao et al. 2009). Briefly, $\mathrm{NaB}$ (PureOne Biotechnology, Shanghai, PRC) was blended into the diet using a food processor at $18 \boldsymbol{g}$ at the proportion of $5 \%$. The NaB-containing diet was pelleted and stored in a $-20^{\circ} \mathrm{C}$ freezer until usage. On the supplemented diet, the mice received $\mathrm{NaB}$ at $5 \mathrm{~g} / \mathrm{kg} /$ day (Gao et al. 2009) at the normal daily rate of caloric intake for a total period of 20 weeks.

Blood glucose levels were recorded on days 0,28 , $56,84,112$ and 140 after the onset of diabetes. Urinary albumin and creatinine were recorded on day 140, after diabetes. The mice were then killed and their kidneys were harvested for analysis.
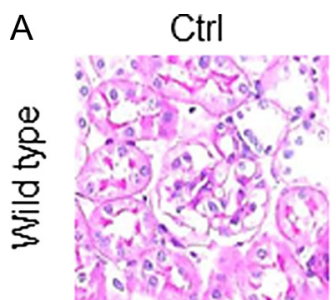

$\mathrm{Ctr} / \mathrm{NaB}$
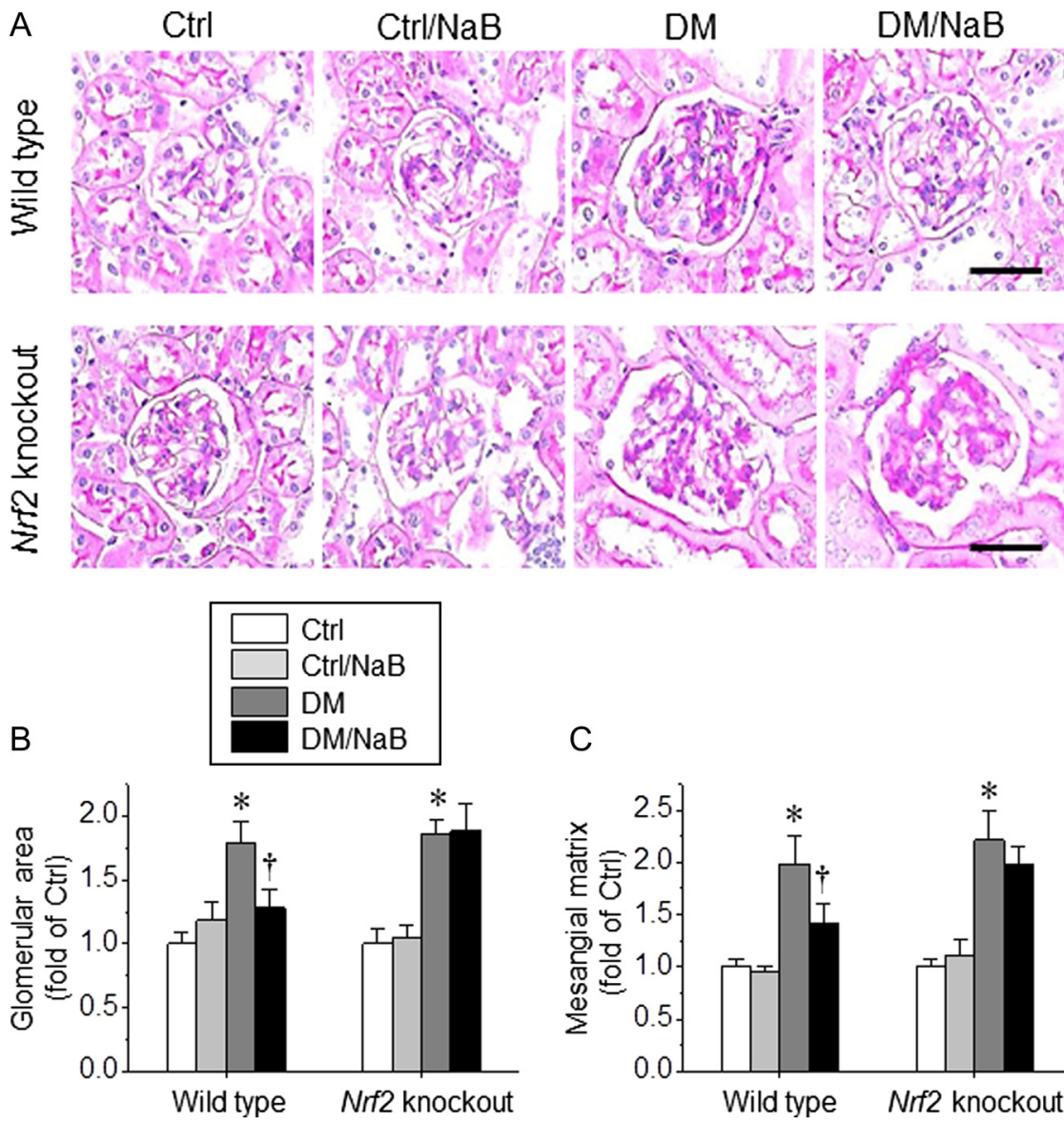

C

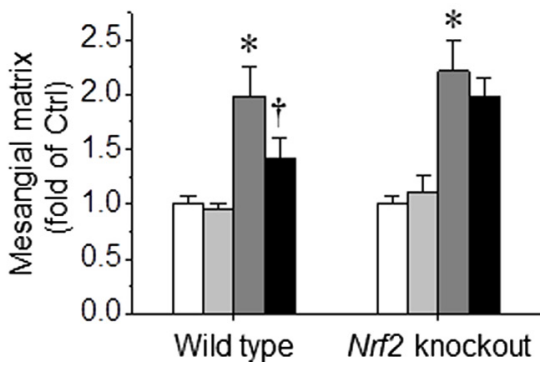

Figure 2

Deletion of the Nrf2 gene completely prevented $\mathrm{NaB}$ protection against the increase in glomerular area and mesangial matrix expansion in diabetic mice. (A) Periodic acid-Schiff (PAS) staining was performed to evaluate renal morphological changes. (B) Glomerular area and (C) mesangial matrix expansion were calculated from PAS staining. Bar $=50 \mu \mathrm{m}$. Data are presented as means \pm s.D. $(n=7)$. ${ }^{*} P<0.05$ vs $C$ trl; ${ }^{+} P<0.05$ vs DM. Bars: white, Ctrl; light grey, $\mathrm{Ctrl} / \mathrm{NaB}$; dark grey, DM; black, DM/NaB. Abbreviations are the same as Fig. 1. A full colour version of this figure is available at http://dx.doi.org/10.1530/ JOE-16-0322. 


\section{Analysis of kidney function}

A mouse albumin ELISA kit (Bethyl Laboratories, Montgomery, TX, USA) and a QuantiChrom Creatinine Assay Kit (BioAssay Systems, Hayward, CA, USA) were used to determine urine albumin and creatinine levels on spot urine sample. Urinary albumin to creatinine ratio (UACR) was calculated.

\section{Western blot analysis}

Western blot analysis was performed using kidney cortex as described in our previous study (Cai et al. 2005). The primary antibodies used were anti-binding immunoglobulin protein (BIP, Cell Signaling, 1:1000),
anti-C/EBP homologous protein (CHOP, Cell Signaling, 1:1000), anti-connective tissue growth factor (CTGF, Santa Cruz Biotechnology, 1:500), anti-glyceraldehyde 3-phosphate dehydrogenase (GAPDH, Santa Cruz Biotechnology, 1:3000), anti-Histone H3 (Santa Cruz Biotechnology, 1:1000), anti-HO1 (Santa Cruz Biotechnology, 1:1000), anti-intercellular adhesion molecule-1 (ICAM-1, Santa Cruz Biotechnology, 1:500), anti-inducible nitric oxide synthase (iNOS, Cell Signaling, 1:1000), anti-KEAP1 (Santa Cruz Biotechnology, 1:500), anti-NQO1 (Santa Cruz Biotechnology, 1:1000), antiNRF2 (Santa Cruz Biotechnology, 1:1000), anti-PAI-1 (Santa Cruz Biotechnology, 1:2000), anti-transforming growth factor beta 1 (TGF- $\beta 1$, Cell Signaling, 1:1000), anti-tumor necrosis factor alpha (TNF- $\alpha$, Cell Signaling,
A

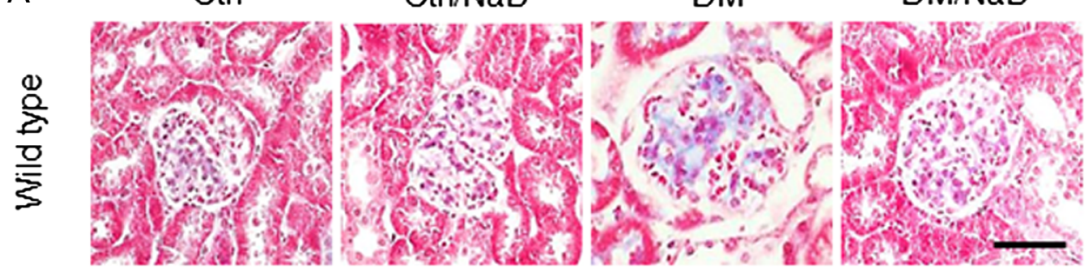

5
$\frac{5}{0}$
$\frac{0}{c}$
$\frac{1}{z}$
$\frac{1}{z}$

Ctrl

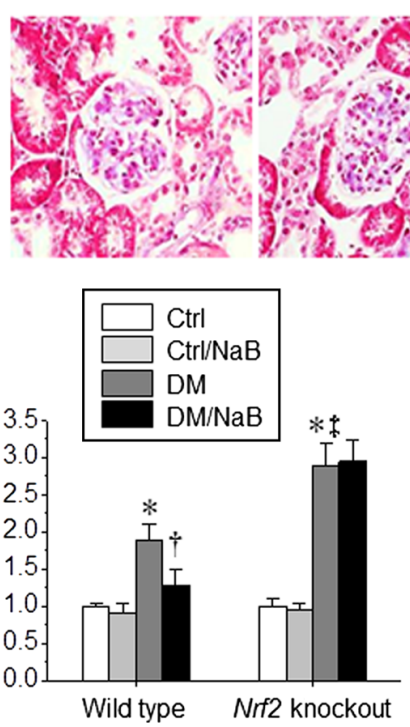

$\mathrm{D}$

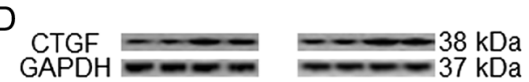

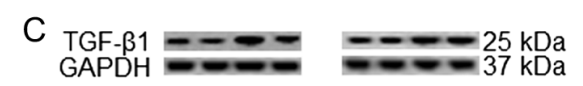

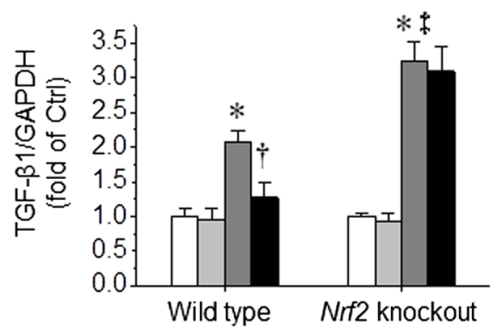

$E$
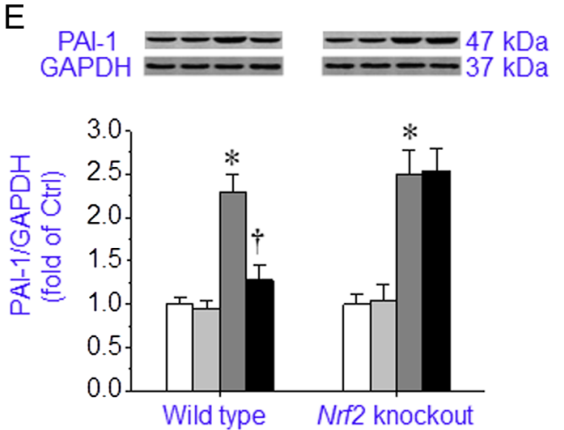

Figure 3

$\mathrm{NaB}$ completely lost its action in preventing from diabetes-induce renal fibrosis in the absence of NRF2. Renal fibrosis was determined by calculating (A) Masson's positive area from Masson's trichrome staining (B). To further evaluate the upstream signaling of fibrosis, (C) transforming growth factor beta 1 (TGF- $\beta 1$ ), (D) connective tissue growth factor (CTGF) and (E) plasminogen activator inhibitor-1 (PAI-1) proteins were determined by Western blot. $B a r=50 \mu \mathrm{m}$. Data are presented as means \pm S.D. $(n=7) .{ }^{*} P<0.05$ vs $\mathrm{Ctrl} ;{ }^{+} P<0.05$ vs $\mathrm{DM} ;{ }^{\ddagger} P<0.05$ vs wild-type DM. Bars: white, Ctrl; light grey, $\mathrm{Ctrl} / \mathrm{NaB}$; dark grey, DM; black, DM/NaB. Abbreviations are the same as Fig. 1. A full colour version of this figure is available at http:// dx.doi.org/10.1530/JOE-16-0322. 
1:500), anti-vascular cell adhesion molecule-1 (VCAM-1, Santa Cruz Biotechnology, 1:500) and anti-3-NT (Millipore, 1:1000).

\section{Real-time PCR}

Quantitative RT-PCR was performed as described in our previous study (Wu et al. 2014). Primers for Nrf2, Ho1, Keap1, Gapdh and Nqo1 were all from Life Technologies.

\section{Histologic staining}

Kidney tissues were fixed immediately in 10\% buffered formalin solution after harvesting, embedded in paraffin and sectioned into 5- $\mu \mathrm{m}$-thick sections onto glass slides. The slides containing the samples were then prepared using periodic acid-Schiff (PAS) and Masson's trichrome staining methods.

\section{Isolation of nuclei}

The renal nuclei were isolated using a nuclei isolation kit (Sigma-Aldrich), as described previously (Wu et al. 2016). Briefly, a kidney cortex (30 mg) from each mouse was homogenized for $45 \mathrm{~s}$ in $150 \mu \mathrm{L}$ of cold lysis buffer containing $0.5 \mu \mathrm{L}$ of dithiothreitol (DTT) and $0.1 \%$ Triton X-100. Post hoc, $300 \mu \mathrm{L}$ of cold $1.8 \mathrm{M}$ Cushion solution (Sucrose Cushion solution:Sucrose Cushion buffer:DTT=900:100:1) was added to the lysis solution. The mixture was then transferred to a new tube preloaded with $150 \mu \mathrm{L}$ of $1.8 \mathrm{M}$ Sucrose Cushion solution followed by centrifugation at $13,000 \mathrm{rpm}$ for $45 \mathrm{~min}$. As a result of the centrifugation, the nuclei were visible as a thin pellet at the bottom of the tube. The supernatant fraction, containing cytosolic components, was kept for analysis of cytosolic NRF2 (c-NRF2).

\section{Immunohistochemical staining}

Immunohistochemical staining was performed, as previously described (Wu et al. 2014), using an antibody against NRF2 (Santa Cruz Biotechnology, 1:100).

\section{Morphometric analyses}

Morphometric analyses were quantified using Image-Pro Plus 6.0 software (Media Cybernetics Inc, Bethesda, MD, USA). Selection of areas to photograph and scoring was done by people blind to the identity of the samples.

\section{Quantitative analysis of lipid peroxides}

Renal malondialdehyde (MDA) concentration was calculated following the instructions by a lipid peroxidation assay kit purchased from Sigma-Aldrich.

\section{HDAC activity assay}

HDAC activity was determined using a HDAC assay kit (BioVision) following the manufacturer's protocol. Briefly, $25 \mu \mathrm{g}$ of renal nuclear extract from each mouse was diluted to $85 \mu \mathrm{L}$ of ddH2O in each well. $10 \mu \mathrm{L}$ of

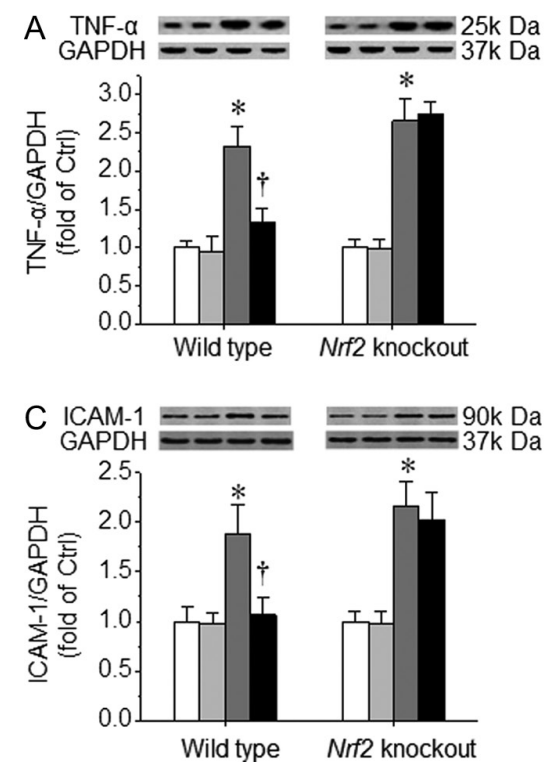

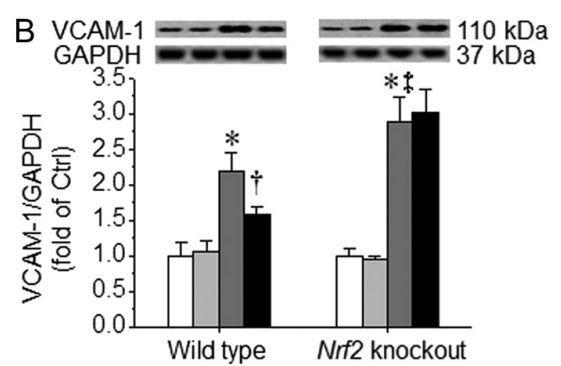

\section{Figure 4}

$\mathrm{NaB}$ protection against diabetes-induced renal inflammation was mediated by NRF2. Renal inflammation was evaluated by measuring protein levels of (A) tumor necrosis factor alpha (TNF- $\alpha$ ), (B) vascular cell adhesion molecule 1 (VCAM-1) and (C) intercellular adhesion molecule 1 (ICAM-1) by Western blot. Data are presented as means \pm S.D. $(n=7) .{ }^{*} P<0.05$ vs $C$ trl; ${ }^{+} P<0.05$ vs DM; ${ }^{\ddagger} P<0.05$ vs wild-type DM. Bars: white, Ctrl; light grey, $\mathrm{Ctrl} / \mathrm{NaB}$; dark grey, DM; black, $\mathrm{DM} / \mathrm{NaB}$. Abbreviations are the same as Fig. 1.
() 2017 Society for Endocrinology Printed in Great Britain 

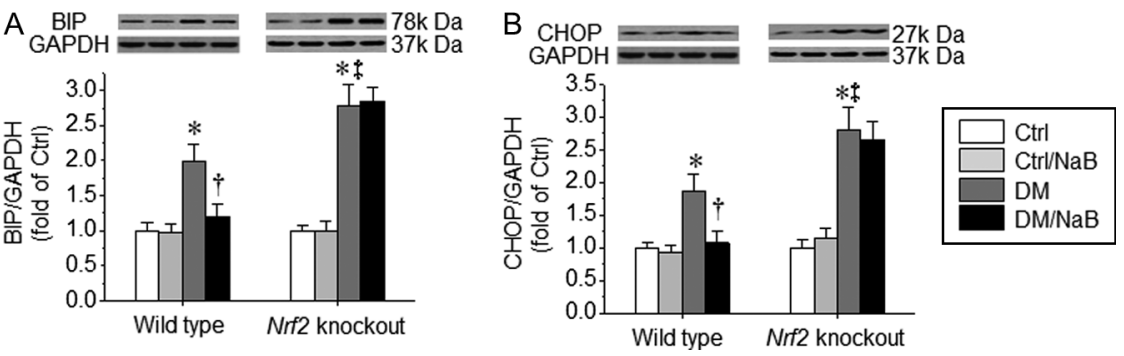

Figure 5

$\mathrm{NaB}$ significantly reduced the expression of renal apoptotic genes, that was induced by diabetes. Renal apoptotic cell death was reflected by protein levels of $(A)$ binding immunoglobulin protein (BIP) and (B) C/EBP homologous protein (CHOP). Data are presented as means \pm S.D. $(n=7)$.

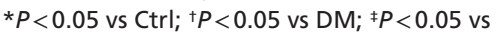
wild-type DM. Bars: white, Ctrl; light grey, $\mathrm{Ctrl} / \mathrm{NaB}$; dark grey, DM; black, DM/NaB. Abbreviations are the same as Fig. 1.

the 10x HDAC assay buffer was added to each well. $5 \mu \mathrm{L}$ of the HDAC fluorometric substrate was added to each well thereafter. After mixing thoroughly, plates were incubated at $37^{\circ} \mathrm{C}$ for $30 \mathrm{~min}$. Reaction was then terminated by adding $10 \mu \mathrm{L}$ of lysine developer, followed by another 30-min incubation.

\section{Statistical analysis}

Seven mice per group were studied. The measurements for each group were summarized as means \pm S.D. Image Quant 5.2 (GE Healthcare Bio-Sciences) was used to analyze Western blots. One-way ANOVA was performed for the comparisons among different groups. The statistical testing was then followed by post hoc pairwise comparisons using Tukey's test with Origin 8.6 data analysis and graphing software (OriginLab, Northampton, MA, USA). A test is considered significant if $P<0.05$.

\section{Results}

\section{Deletion of the Nrf2 gene completely abolished $\mathrm{NaB}$ protection against diabetes-induced renal dysfunction}

Diabetic mice developed significantly higher blood glucose levels from 0 to 20 weeks after the onset of diabetes in both wild-type (Fig. 1A) and Nrf2-knockout (Fig. 1B) mice. NaB did not alter blood glucose levels under both diabetic and non-diabetic conditions in both types of mice (Fig. 1A and B). In wild-type mice, $\mathrm{NaB}$ significantly reduced the ratio of kidney weight to tibia length which was, in turn, increased by diabetes (Fig. 1C, left panel). However, $\mathrm{NaB}$ completely lost this action in Nrf2-knockout mice (Fig. 1C, right panel). UACR, which indicates renal function, was determined in all mice (Fig. 1D). Both wild-type and Nrf2-knockout diabetic mice had significantly higher UACRs as compared to their relative controls (Fig. 1D). Moreover, deletion of the Nrf2 gene
A

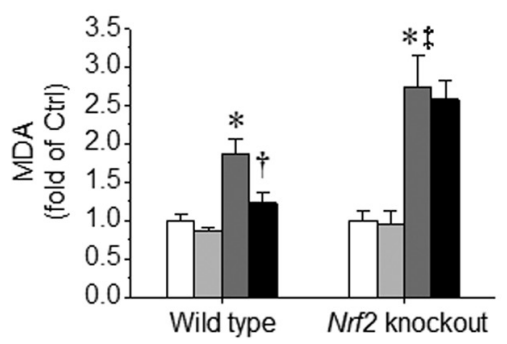

C

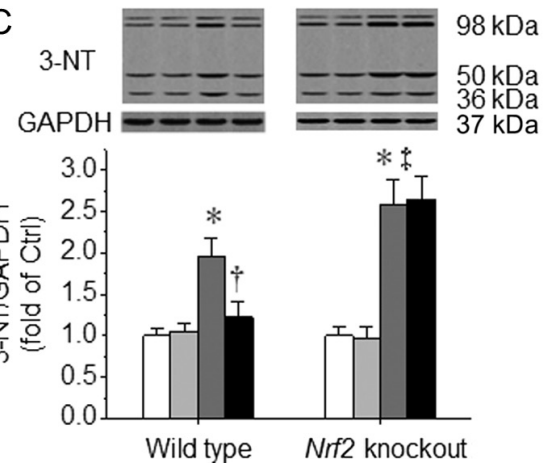

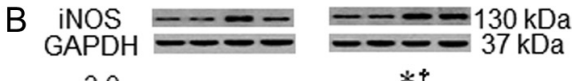

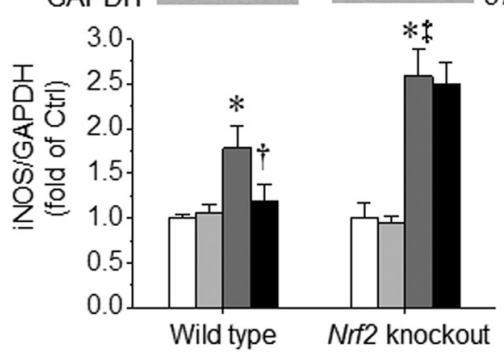

Figure 6

NRF2 played a key role in $\mathrm{NaB}$ amelioration of diabetes-induced renal oxidative damage. Renal (A) malondialdehyde (MDA) concentration was calculated by a lipid peroxidation assay kit. (B) Inducible nitric oxide synthase (iNOS) and 3-Nitrotyrosine (3-NT) proteins were measured by Western blot. Data are presented as means \pm S.D. $(n=7) .{ }^{*} P<0.05$ vs $C$ trl; ${ }^{+} P<0.05$ vs DM; ${ }^{\ddagger} P<0.05$ vs wild-type DM. Bars: white, Ctrl; light grey, $\mathrm{Ctrl} / \mathrm{NaB}$; dark grey, DM; black, DM/NaB. Abbreviations are the same as Fig. 1. 

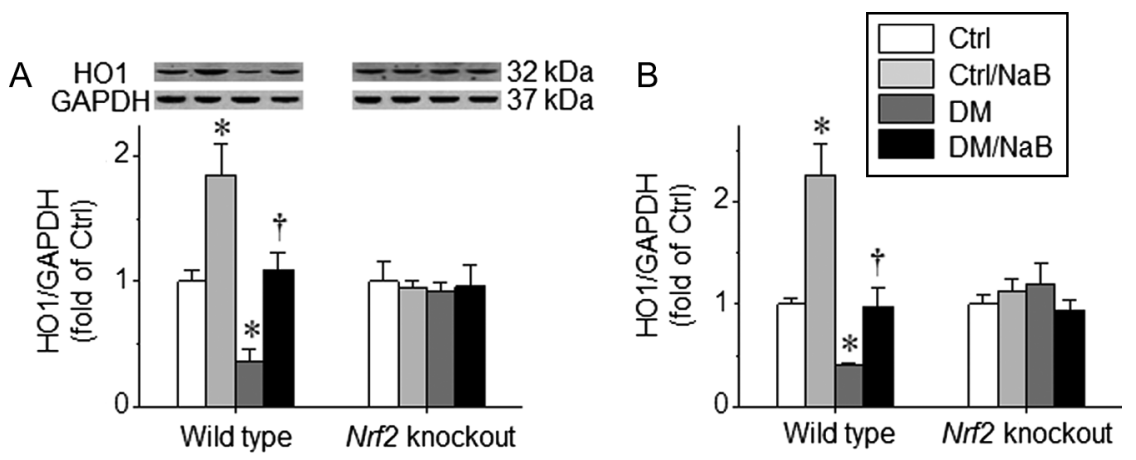

C
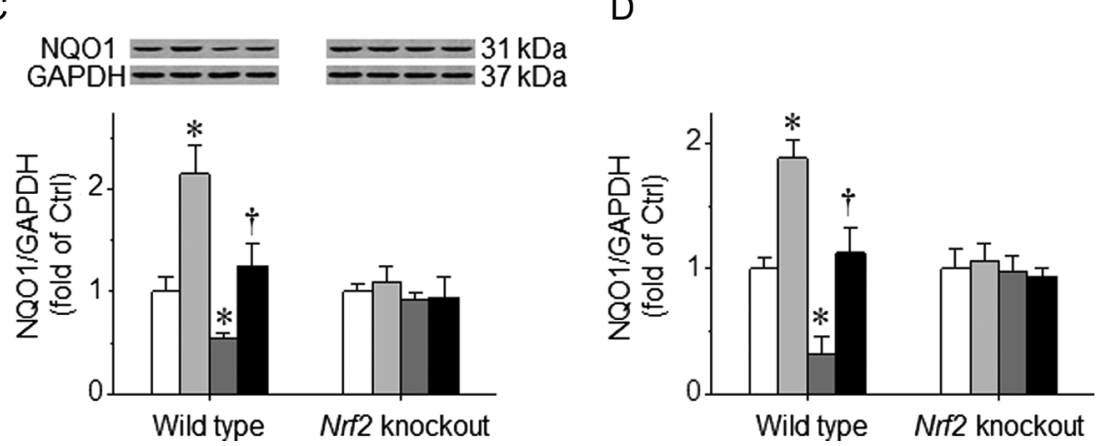

Figure 7

$\mathrm{NaB}$ increased the expression of $\mathrm{Nrf2}$ downstream antioxidant genes. Heme oxygenase 1 (Ho1) (A) protein and (B) mRNA, as well as $\mathrm{NAD}(\mathrm{P}) \mathrm{H}$ dehydrogenase quinone (NqO1) (C) protein and (D) mRNA were all determined to evaluate NRF2 function as a transcription factor for its downstream targets. resulted in more severer diabetes-induced renal dysfunction compared with wild-type diabetic mice (Fig. 1D), which was in accordance with our previous studies (Wu et al. 2015b, 2016). Notably, NaB markedly decreased UACR that was elevated by diabetes in wildtype mice (Fig. 1D, left panel). However, the effect of $\mathrm{NaB}$ was completely abolished in the absence of Nrf2 (Fig. 1D, right panel). These results indicate that NRF2 plays a key role in $\mathrm{NaB}$ protection against diabetes-induced renal dysfunction.

\section{Deletion of the Nrf2 gene completely prevented NaB protection against the increase in glomerular area and mesangial matrix expansion in diabetic mice}

To test the effect of $\mathrm{NaB}$ on diabetes-induced renal pathological changes in wild-type and Nrf2-knockout mice, PAS staining was performed (Fig. 2A). Both wild-type and Nrf2-knockout diabetic mice had a significant increase in glomerular area and mesangial matrix expansion as compared to relative controls (Fig. 2B and C). Notably, $\mathrm{NaB}$ showed this efficacy in wild-type mice (Fig. 2B and C, left panels), but not in Nrf2-knockout mice (Fig. 2B and $\mathrm{C}$, right panels). These results suggest that NRF2 is required for $\mathrm{NaB}$ protection against diabetes-induced increase in glomerular area and mesangial matrix expansion.
$\mathrm{NaB}$ completely lost its action in preventing from diabetes-induced renal fibrosis in the absence of NRF2

Next, we determined renal fibrosis in all mice. Thus, Masson's trichrome staining was performed (Fig. 3A) with Masson's positive area calculated (Fig. 3B). Protein levels of TGF- $\beta 1$ (Fig. 3C), CTGF (Fig. 3D) and PAI-1 (Fig. 3E) were determined by Western blot. NaB significantly prevented from diabetes-enhanced renal Masson's positive area, TGF- $\beta 1$, CTGF and PAI- 1 in wild-type mice (Fig. 3B, C, D and E, left panels). However, deletion of the Nrf2 gene completely blocked the actions of $\mathrm{NaB}$ (Fig. 3B, C, D and E, right panels). Therefore, NRF2 is the key factor through which $\mathrm{NaB}$ prevents diabetic renal fibrosis.

\section{$\mathrm{NaB}$ prevention of diabetes-induced renal inflammation was mediated by NRF2}

Renal inflammation was determined by Western blot analysis of TNF- $\alpha$ (Fig. 4A), VCAM-1 (Fig. 4B) and ICAM-1 (Fig. 4C). Diabetes produced renal inflammation in both types of mice (Fig. 4A, B and C). Wild-type mice, but not Nrf2-knockout mice, were prevented from diabetesinduced renal inflammation by $\mathrm{NaB}$ (Fig. 4A, B and C). Therefore, the results of the mechanism suggest that $\mathrm{NaB}$ prevention of diabetes-induced renal inflammation is mediated by NRF2.

Published by Bioscientifica Ltd 


\section{$\mathrm{NaB}$ significantly reduced the expression of renal apoptotic genes that was induced by diabetes}

Renal apoptotic gene expression was tested by measuring protein levels of BIP (Fig. 5A) and CHOP (Fig. 5B). Diabetic kidney exihibited increased BIP and $\mathrm{CHOP}$ proteins, and this effect was almost completely prevented by administration of $\mathrm{NaB}$ (Fig. 5A and B, left panels). In contrast, Nrf2 gene deletion resulted in a complete abolishment of this protective effect of $\mathrm{NaB}$ (Fig. 5A and $\mathrm{B}$, right panels). These findings indicate that $\mathrm{NaB}$ has the capacity to prevent from diabetes-induced expression of renal apoptotic genes.

\section{NRF2 played a key role in NaB amelioration of diabetes-induced renal oxidative damage}

Because NRF2 is the governor of the cellular antioxidant system (Kesic et al. 2011, Pendyala et al. 2011, Ruiz et al. 2013), it is important to test renal oxidative damage. MDA, iNOS and 3-NT, as indicators of oxidative damage (Sari et al. 2014), were determined in all mice (Fig. 6A, $\mathrm{B}$ and $\mathrm{C})$. Diabetes significantly elevated renal MDA, iNOS and 3-NT in both types of mice (Fig. 6A, B and C). Furthermore, Nrf2 gene deletion led to a significant increase in MDA, iNOS and 3-NT compared with wildtype mice (Fig. 6A, B and C). Moreover, NaB markedly
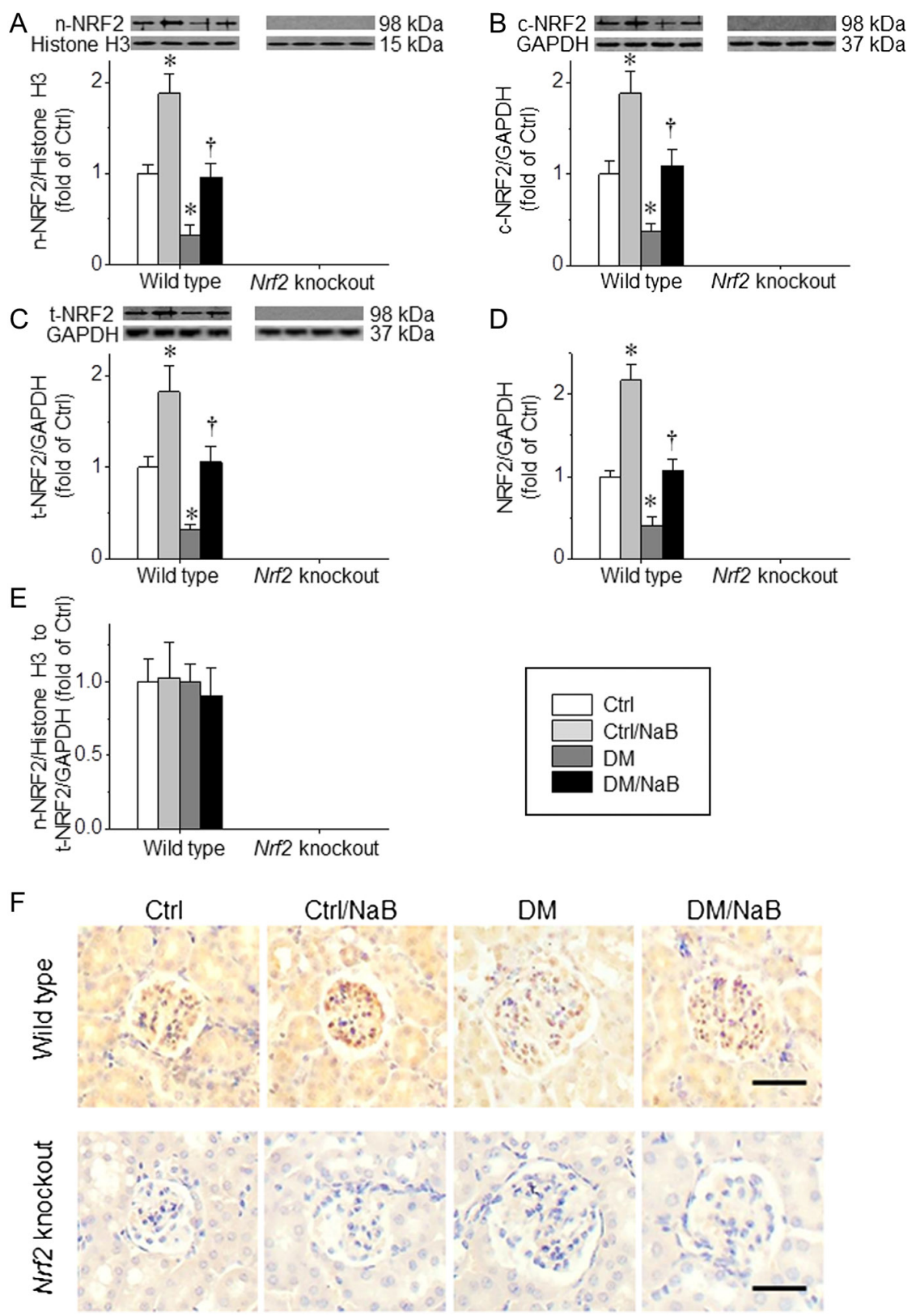

\section{Figure 8}

$\mathrm{NaB}$ upregulated $\mathrm{Nrf2}$ expression without increasing the proportion of NRF2 nuclear translocation. Western blot assays were performed to determine (A) nuclear NRF2 (n-NRF2), (B) cytosolic NRF2 (c-NRF2) and (C) total NRF2 (t-NRF2). To test the effect of $\mathrm{NaB}$ on Nrf2 gene transcription, (D) NRF2 mRNA was determined by RT-PCR. To further investigate the effect of NaB on NRF2 translocation, (E) a comparison of the ratios of $n-N R F 2 / H i s t i o n e ~ H 3$ to t-NRF2/GAPDH between the four groups was constructed. (F) Immunohistochemical staining was performed to observe the location of NRF2 expression in the kidneys of all mice. Data are presented as means \pm s.D. $(n=7) . * P<0.05$ vs $C \operatorname{trl} ;$ ${ }^{+} P<0.05$ vs DM. Bars: white, Ctrl; light grey, Ctrl/ $\mathrm{NaB}$; dark grey, DM; black, DM/NaB.

Abbreviations are the same as Fig. 1. A full colour version of this figure is available at http://dx.doi. org/10.1530/JOE-16-0322. http://joe.endocrinology-journals.org DOI: 10.1530/JOE-16-0322
๑) 2017 Society for Endocrinology Printed in Great Britain 
decreased these levels in the kidneys of wild-type diabetic mice, but not Nrf2-knockout diabetic mice (Fig. 6A, B and $\mathrm{C})$. These results, on one hand, confirmed the critical role of NRF2 in the prevention of diabetic renal oxidative damage and addressed the importance of NRF2 in NaB renal protective function in $\mathrm{DN}$, on the other.

\section{$\mathrm{NaB}$ increased the expression of $\mathrm{Nrf2}$ downstream antioxidant genes}

To evaluate NRF2 function, the expression of $\mathrm{Ho} 1$ and Nqo1, targets of NRF2 (Martin et al. 2004, He et al. 2009), was determined by Western blot and RT-PCR. In wild-type mice, NaB markedly elevated the expression of Ho1 and Nqo1 under both diabetic and non-diabetic conditions (Fig. 7A, B, C and D, left panels). However, the efficacies of $\mathrm{NaB}$ were completely blocked in Nrf2-knockout mice (Fig. 7A, B, C and D, right panels). Hence, NRF2 is the key factor through which $\mathrm{NaB}$ upregulates the expression of renal antioxidant genes.

\section{NaB upregulated $N r f 2$ expression without increasing the proportion of NRF2 nuclear translocation}

As NRF2 is a transcription factor, it is important to measure the amount of NRF2 in the nucleus (nuclear NRF2, n-NRF2), which also reflects NRF2 function. NaB significantly increased n-NRF2 under both diabetic and non-diabetic conditions in wild-type mice (Fig. 8A, left panel). Similarly, NRF2 in the cytoplasm (c-NRF2), whole cell (total NRF2,
t-NRF2) and Nrf2 mRNA were all increased by $\mathrm{NaB}$ (Fig. 8B, $\mathrm{C}$ and D, left panels). However, $\mathrm{Nrf2}$ protein and mRNA were not detectable in Nrf2-knockout mice (Fig. 8A, B, C and D, right panels), and this comfirmed the deletion of the $\mathrm{Nrf2}$ gene. To investigate whether NaB promotes NRF2 nuclear translocation, we further constructed a comparison of the ratios of n-NRF2/Histione H3 to t-NRF2/GAPDH between the four groups (Fig. 8E). Hypothetically, this ratio could reflect the proportion of the nuclear translocated NRF2 in total NRF2. $\mathrm{NaB}$ did not alter the proportion of nuclear translocated NRF2 under either diabetic or non-diabetic conditions in wild-type mice (Fig. 8E, left panel). Hence, $\mathrm{NaB}$ may have no impact on NRF2 nuclear translocation. The fact that $\mathrm{NaB}$ increased NRF2 mRNA and did not promote NRF2 translocation to the nucleus sheds light on the regulation of NRF2 by NaB. Thus, the results indicate that this mechanism occurred predominantly at the transcriptional level. Immunohistochemical staining was performed to show the localization of NRF2 (Fig. 8F), which was observed preferably in the glomeruli of wildtype mice (Fig. 8F, upper panel). Nrf2-knockout kidney did not show a positive staining of NRF2 (Fig. 8F, lower panel).

\section{KEAP1 was not involved in NaB regulation of NRF2 function}

KEAP1 is known to sequester NRF2 in the cytoplasm, restricting NRF2 from nuclear translocation and facilitating NRF2 ubiquitination (Miyata et al. 2013). KEAP1 protein and mRNA were elevated in both
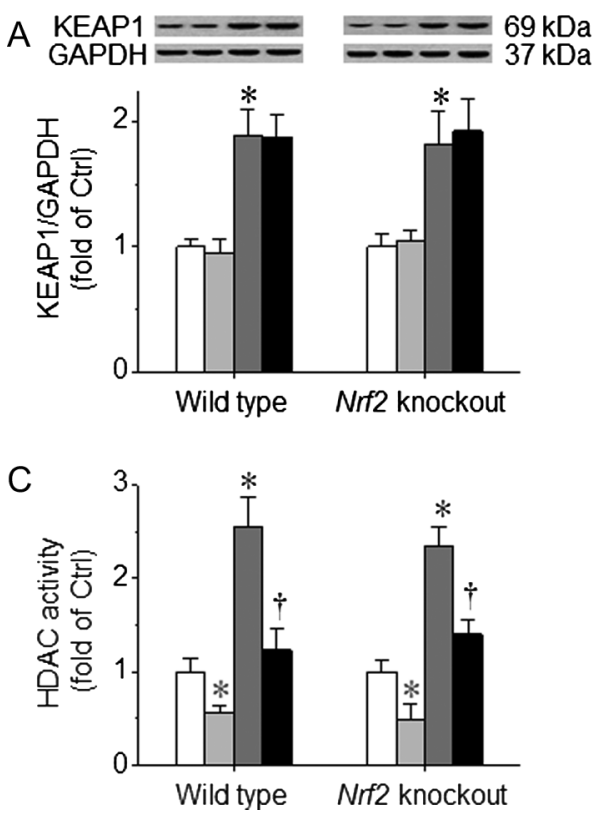

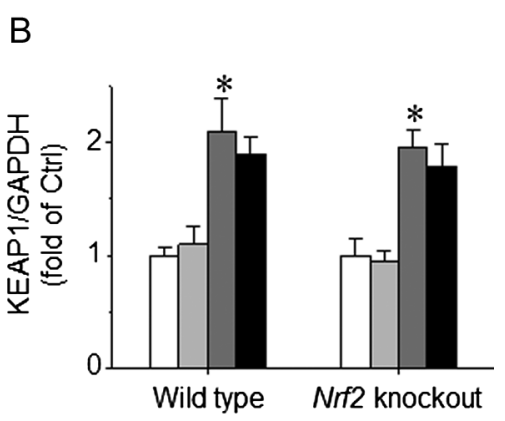

Figure 9

$\mathrm{NaB}$ did not alter renal Kelch-like ECH-associated protein 1 (Keap1) expression, while significantly inhibited the activity of histone deacetylase (HDAC). We further determined renal Keap1 (A) protein and (B) mRNA, along with total HDAC activity in all mice. Data are presented as means \pm s.D. $(n=7)$. ${ }^{\star} P<0.05$ vs $\mathrm{Ctrl} ;{ }^{+} P<0.05$ vs DM. Bars: white, Ctrl; light grey, $\mathrm{Ctrl} / \mathrm{NaB}$; dark grey, DM; black, DM/NaB. Abbreviations are the same as Fig. 1. 
wild-type and Nrf2-knockout diabetic mice (Fig. 9A and B). However, NaB showed no impact on Keap1 expression in the two types of mice under both diabetic and non-diabetic conditions (Fig. 9A and B). Therefore, KEAP1 was not involved in NaB regulation of NRF2 expression and function. This result is in accordance with the finding that $\mathrm{NaB}$ did not promote nuclear translocation of NRF2 (Fig. 8E).

\section{$\mathrm{NaB}$ inhibited HDAC activity in the kidneys of both wild-type and Nrf2-knockout mice}

$\mathrm{NaB}$ is an inhibitor of HDAC activity. Therefore, the effect of HDAC inhibition by $\mathrm{NaB}$ may facilitate transcription factors to bind the promotor region of the Nrf2 gene, which may concequently lead to Nrf2 gene transcription. To further confirm the inhibitory effect of $\mathrm{NaB}$ on HDAC activity, we determined the total HDAC activity in all mice. As shown in Fig. 9C, HDAC activity was markedly inhibited by $\mathrm{NaB}$ in both wild-type and Nrf2-knockout mice, under both nondiabetic and diabetic conditions. Thus, the activation of $\mathrm{Nrf2}$ transcription (Fig. 8D) may be facilitated by $\mathrm{NaB}$ inhibition of HDAC activity.

\section{Discussion}

The present study explored the mechanism by which $\mathrm{NaB}$ ameliorates $\mathrm{DN}$ in mice. $\mathrm{NaB}$ showed a promising preventive effect on DN in C57BL/6 wild-type mice. By using Nrf2-knockout mice, we identified NRF2 to be the key factor through, which $\mathrm{NaB}$ ameliorated DN. Given that Keap1 expression and NRF2 nuclear translocation were not altered by $\mathrm{NaB}$, the present study indicates that $\mathrm{NaB}$ may upregulate Nrf2 expression and function at transcriptional level. This action may be facilitated by the inhibitory effect of $\mathrm{NaB}$ on HDAC activity (Fig. 10).

Although $\mathrm{NaB}$ was found be beneficial to several diseases (He et al. 2016, Li et al. 2016, Salimi et al. 2016, Subramanian et al. 2016), little is known about its protection against DN. To the best of our knowledge, there has been only one study that reported $\mathrm{NaB}$ protection against DN (Khan \& Jena 2014). The study by Khan and coworkers showed that $\mathrm{NaB}$ ameliorated oxidative damage and fibrosis in the kidneys of diabetic rats along with a decrease in HDAC activity (Khan \& Jena 2014). However, given that HDAC inhibition could have a positive effect on transcription of a variety of genes, it is not certain which gene activation plays a key role

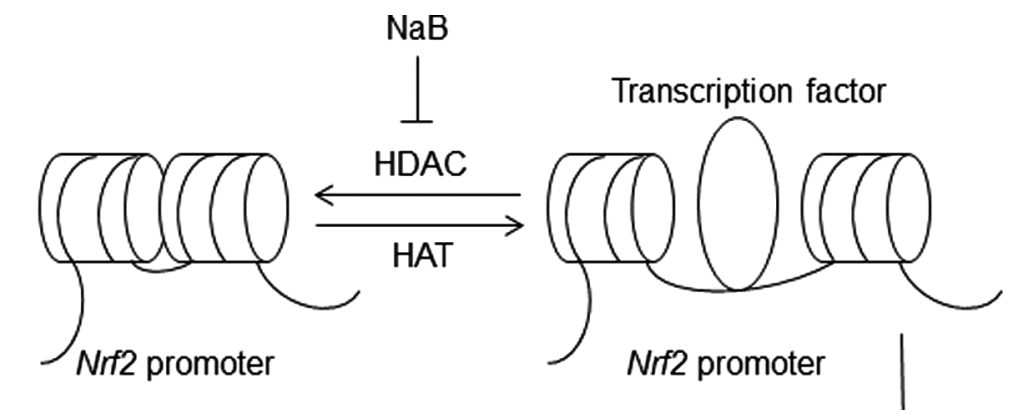

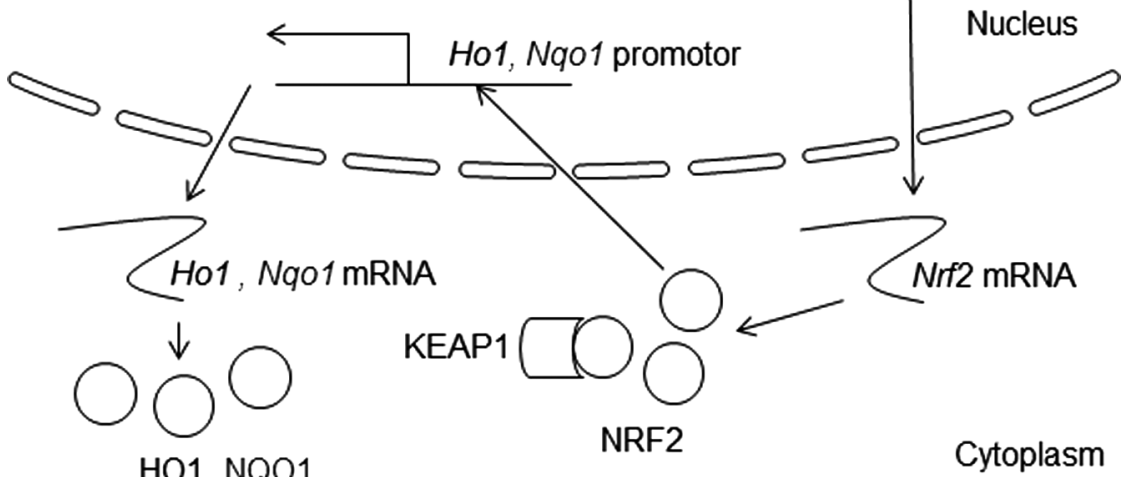
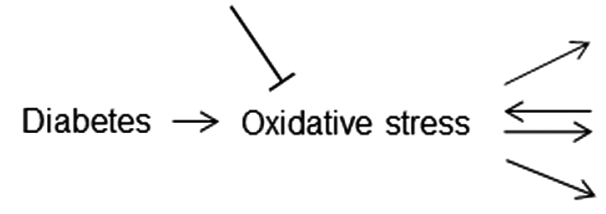

Apoptosis

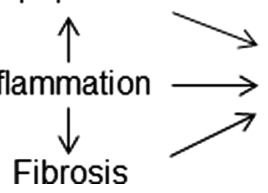

\section{Figure 10}

Possible mechanism by which $\mathrm{NaB}$ ameliorated DN. NaB inhibited the activity of HDAC, opening the structure of chromatin. This effect could facilitate transcription factor to bind the promoter region of $\mathrm{Nrf2}$ gene, followed by increased $\mathrm{Nrf2}$ gene expression. Keap1 expression and NRF2 nuclear translocation were not altered by $\mathrm{NaB}$, indicating that excessive cytosolic NRF2, in turn, might enter the nucleus and turned on the transcription of its downstream targets $\mathrm{Ho} 1$ and $\mathrm{NqO1}$. As a result, antioxidants, such as $\mathrm{HO}$ and NQO1, were increased, and this blocked oxidative damage caused by diabetes, the effect of which ameliorated diabetic renal inflammation, fibrosis, apoptosis and eventually typical hallmarks of diabetic nephropathy. $\downarrow$, activation; $\perp$, inhibition. 
in the protective effect of $\mathrm{NaB}$. In the present study, we observed the activation of $\mathrm{Nrf2}$ gene expression by $\mathrm{NaB}$ (Fig. 8A, B, C and D, left panels), along with increased expression of NRF2's downstream targets $\mathrm{Ho1}$ and Nqo1 (Fig. 7A, B, C and D, left panels). Additionally, NaB ameliorated diabetes-induced renal oxidative damage, apoptosis, inflammation, fibrosis, pathological changes and renal dysfunction. Notably, these actions of $\mathrm{NaB}$ were completely abolished in the absence of Nrf2. Therefore, the results demonstrate that the activation of Nrf2, but not other genes, plays a key role in $\mathrm{NaB}$ protection against DN. The mechanism by which NaB-activated Nrf2 transcription may be its inhibitory effect on HDAC activity (Fig. 9C). The result of the inhibition could, in turn, facilitate transcription factors to bind the promoter region of the Nrf2 gene furthering its gene transcription (Fig. 10). Although $\mathrm{NaB}$ may facilitate the transcription of many genes by the inhibition of HDAC, the activation of Nrf2 gene transcription provided the predominant benefit in $\mathrm{NaB}$ protection against $\mathrm{DN}$. Supporting this notion, SFN, another HDAC inhibitor, completely lost its function in ameliorating DN in the absence of NRF2 (Wu et al. 2015b). This indicates that NRF2 is the key factor in both $\mathrm{NaB}$ and SFN protection against $\mathrm{DN}$.

KEAP1 sequesters NRF2 in the cytoplasm, promoting its proteasomal degradation (Miyata et al. 2013). Thus, application of small molecules that can either modify the structure of KEAP1 to release NRF2 or reduce cytoplasmic KEAP1 can be used to activate NRF2 function. SFN modifies specific cysteine residues in KEAP1, enabling NRF2 to escape from KEAP1-mediated ubiquitination and degradation (Zhang \& Hannink 2003). As a result, NRF2 translocates into the nucleus to activate transcription of downstream antioxidant enzymes (Zhang \& Hannink 2003, Hu et al. 2011). Curcumin is also known as an activator of NRF2. Like SFN, curcumin disrupts the binding between KEAP1 and NRF2, leading to the release of NRF2 (Esatbeyoglu et al. 2012). However, the fact that curcumin downregulates Keap1 expression (Soetikno et al. 2013) challenges the notion that curcumin only modifies the KEAP1-NRF2 complex. In our previous study, we demonstrated that the curcumin analog, C66, decreased Keap1 mRNA and protein via the upregulation of microRNA-200a (Wu et al. 2016). Therefore, in addition to disruption of KEAP1-NRF2 complex, the decreased expression of Keap1 may also account for NRF2 induction by curcumin (Wu et al. 2016). Nevertheless, unlike SFN, curcumin and C66, NaB had no impact on Keap1 expression (Fig. 9A and B) and NRF2 nuclear translocation (Fig. 8E) under both diabetic and non-diabetic conditions.
The present study indicates a different regulatory mechanism of Nrf2 expression by $\mathrm{NaB}$ from that by SFN, curcumin and C66. We, therefore, speculate that $\mathrm{NaB}$ treatment produced excessive NRF2 (Fig. 8A, B and C, left panels) that surpassed the neutralizing ability of KEAP1. Hence, $\mathrm{NaB}$ might solely regulate $\mathrm{Nrf2}$ expression at the transcriptional level, which may be facilitated by the inhibition of HDAC activity (Fig. 10).

Both NRF2 activators and NaB have been applied in clinical trials. Although bardoxolone methyl, a NRF2 activator, was terminated in a phase III study due to heart complications (Hall \& Bhalla 2014), NRF2 remains a viable drug target as evidenced by the approval of BG-12 (dimethyl fumarate) in treatment of multiple sclerosis (Gold et al. 2012). NaB has also been used in clinical trials. $\mathrm{NaB}$ improved the efficacy of oral mesalazine in active ulcerative colitis (Vernia et al. 2000); reduced inflammation in stool of the patients with shigellosis, along with an improvement in rectal histopathology (Raqib et al. 2012); reduced the frequency of abdominal pain in patients with irritable bowel syndrome (Banasiewicz et al. 2013) and reduced the frequency of diverticulitis episodes (Krokowicz et al. 2014). Thus, there is a potential use of NaB, the NRF2 activator, in the prevention of DN. Although evidence shows that $\mathrm{NaB}$ functioned predominantly through the activation of NRF2 in the mouse model of this study, more animal studies are needed before the potential application of $\mathrm{NaB}$ in humans.

Taken together, the present study demonstrates, for the first time, that NRF2 plays a key role in $\mathrm{NaB}$ protection against DN. Furthermore, this study indicates that $\mathrm{NaB}$ regulates $\mathrm{Nrf2}$ expression at the transcriptional level, possibly through the inhibition of HDAC activity. Therefore, the present study may provide a basis for the future study or application of $\mathrm{NaB}$.

\section{Declaration of interest}

The authors declare that there is no conflict of interest that could be perceived as prejudicing the impartiality of the research reported.

\section{Funding}

This work was supported in part by Norman Bethune Program of Jilin University (2015438) and National Natural Science Foundation of China (81600573) to H W; National Natural Science Foundation of China Grant (81400725), Jilin University Bethune Foundation Grant (2015201) and Natural Science Foundation of Jilin Province (20160101030JC) to W S; and National Basic Research Program of China (2014CB543100) to F W.

\section{Acknowledgements}

The authors thank Tanya B Hanson for proofreading this article. 


\section{References}

Banasiewicz T, Krokowicz L, Stojcev Z, Kaczmarek BF, Kaczmarek E, Maik J, Marciniak R, Krokowicz P, Walkowiak J \& Drews M 2013 Microencapsulated sodium butyrate reduces the frequency of abdominal pain in patients with irritable bowel syndrome. Colorectal Disease 15 204-209. (doi:10.1111/j.1463-1318.2012.03152.x)

Cai L, Wang J, Li Y, Sun X, Wang L, Zhou Z \& Kang YJ 2005 Inhibition of superoxide generation and associated nitrosative damage is involved in metallothionein prevention of diabetic cardiomyopathy. Diabetes $\mathbf{5 4}$ 1829-1837. (doi:10.2337/diabetes.54.6.1829)

de Haan JB 2011 Nrf2 activators as attractive therapeutics for diabetic nephropathy. Diabetes 60 2683-2684. (doi:10.2337/db11-1072)

Dronavalli S, Duka I \& Bakris GL 2008 The pathogenesis of diabetic nephropathy. Nature Clinical Practice Endocrinology and Metabolism 4 444-452. (doi:10.1038/ncpendmet0894)

Esatbeyoglu T, Huebbe P, Ernst IM, Chin D, Wagner AE \& Rimbach G 2012 Curcumin - from molecule to biological function. Angewandte Chemie International Edition in English 51 5308-5332. (doi:10.1002/ anie.201107724)

Gao Z, Yin J, Zhang J, Ward RE, Martin RJ, Lefevre M, Cefalu WT \& Ye J 2009 Butyrate improves insulin sensitivity and increases energy expenditure in mice. Diabetes 58 1509-1517. (doi:10.2337/db081637)

Gold R, Kappos L, Arnold DL, Bar-Or A, Giovannoni G, Selmaj K, Tornatore C, Sweetser MT, Yang M, Sheikh SI, et al. 2012 Placebo-controlled phase 3 study of oral BG-12 for relapsing multiple sclerosis. New England Journal of Medicine 367 1098-1107. (doi:10.1056/NEJMoa1114287)

Hall ET \& Bhalla V 2014 Is there a sweet spot for Nrf2 activation in the treatment of diabetic kidney disease? Diabetes 63 2904-2905. (doi:10.2337/db14-0829)

He X, Kan H, Cai L \& Ma Q 2009 Nrf2 is critical in defense against high glucose-induced oxidative damage in cardiomyocytes. Journal of Molecular and Cellular Cardiology 46 47-58. (doi:10.1016/j. yjmcc.2008.10.007)

He B, Wang M, Guo H, Jia Y, Yang X \& Zhao R 2016 Effects of sodium butyrate supplementation on reproductive performance and colostrum composition in gilts. Animal 10 1722-1727. (doi:10.1017/ S1751731116001269)

Hu C, Eggler AL, Mesecar AD \& van Breemen RB 2011 Modification of keap1 cysteine residues by sulforaphane. Chemical Research in Toxicology 24 515-521. (doi:10.1021/tx100389r)

Keshari KR, Wilson DM, Sai V, Bok R, Jen KY, Larson P, Van Criekinge M, Kurhanewicz J \& Wang ZJ 2014 Non-invasive in vivo imaging of diabetes-induced renal oxidative stress and response to therapy using hyperpolarized 13C dehydroascorbate magnetic resonance. Diabetes 64 344-352. (doi:10.2337/db13-1829)

Kesic MJ, Simmons SO, Bauer R \& Jaspers I 2011 Nrf2 expression modifies influenza A entry and replication in nasal epithelial cells. Free Radical Biology and Medicine 51 444-453. (doi:10.1016/j. freeradbiomed.2011.04.027)

Khan S \& Jena G 2014 Sodium butyrate, a HDAC inhibitor ameliorates eNOS, iNOS and TGF-beta1-induced fibrogenesis, apoptosis and DNA damage in the kidney of juvenile diabetic rats. Food and Chemical Toxicology 73 127-139. (doi:10.1016/j.fct.2014.08.010)

Krokowicz L, Stojcev Z, Kaczmarek BF, Kociemba W, Kaczmarek E, Walkowiak J, Krokowicz P, Drews M \& Banasiewicz T 2014 Microencapsulated sodium butyrate administered to patients with diverticulosis decreases incidence of diverticulitis - a prospective randomized study. International Journal of Colorectal Disease $\mathbf{2 9}$ 387-393. (doi:10.1007/s00384-013-1807-5)

Li H, Sun J, Wang F, Ding G, Chen W, Fang R, Yao Y, Pang M, Lu ZQ \& Liu J 2016 Sodium butyrate exerts neuroprotective effects by restoring the blood-brain barrier in traumatic brain injury mice. Brain Research 1642 70-78. (doi:10.1016/j.brainres.2016.03.031)
Liu H, Zhang JJ, Li X, Yang Y, Xie XF \& Hu K 2015 Post-occlusion administration of sodium butyrate attenuates cognitive impairment in a rat model of chronic cerebral hypoperfusion. Pharmacology Biochemistry and Behavior 135 53-59. (doi:10.1016/j. pbb.2015.05.012)

Martin D, Rojo AI, Salinas M, Diaz R, Gallardo G, Alam J, De Galarreta CM \& Cuadrado A 2004 Regulation of heme oxygenase-1 expression through the phosphatidylinositol 3-kinase/Akt pathway and the Nrf2 transcription factor in response to the antioxidant phytochemical carnosol. Journal of Biological Chemistry 279 8919-8929. (doi:10.1074/jbc. M309660200)

Miyata T, Suzuki N \& van Ypersele de Strihou C 2013 Diabetic nephropathy: are there new and potentially promising therapies targeting oxygen biology? Kidney International 84 693-702. (doi:10.1038/ki.2013.74)

Pendyala S, Moitra J, Kalari S, Kleeberger SR, Zhao Y, Reddy SP, Garcia JG \& Natarajan V 2011 Nrf2 regulates hyperoxia-induced Nox4 expression in human lung endothelium: identification of functional antioxidant response elements on the Nox4 promoter Free Radical Biology and Medicine 50 1749-1759. (doi:10.1016/j. freeradbiomed.2011.03.022)

Raqib R, Sarker P, Mily A, Alam NH, Arifuzzaman AS, Rekha RS, Andersson J, Gudmundsson GH, Cravioto A \& Agerberth B 2012 Efficacy of sodium butyrate adjunct therapy in shigellosis: a randomized, double-blind, placebo-controlled clinical trial. BMC Infectious Diseases 12 111. (doi:10.1186/1471-2334-12-111)

Ruiz S, Pergola PE, Zager RA \& Vaziri ND 2013 Targeting the transcription factor Nrf2 to ameliorate oxidative stress and inflammation in chronic kidney disease. Kidney International 83 1029-1041. (doi:10.1038/ki.2012.439)

Salimi V, Shabani M, Nourbakhsh M \& Tavakoli-Yaraki M 2016 Involvement of 15-lipoxygenase-1 in the regulation of breast cancer cell death induced by sodium butyrate. Cytotechnology 68 2519-2528. (doi:10.1007/s10616-016-9972-3)

Sari AN, Kacan M, Unsal D, Sahan Firat S, Kemal Buharalioglu C, Vezir O, Korkmaz B, Cuez T, Canacankatan N, Sucu N, et al. 2014 Contribution of RhoA/Rho-kinase/MEK1/ERK1/2/iNOS pathway to ischemia/reperfusion-induced oxidative/nitrosative stress and inflammation leading to distant and target organ injury in rats. European Journal of Pharmacology $\mathbf{7 2 3}$ 234-245. (doi:10.1016/j. ejphar.2013.11.027)

Soetikno V, Sari FR, Lakshmanan AP, Arumugam S, Harima M, Suzuki K, Kawachi H \& Watanabe K 2013 Curcumin alleviates oxidative stress, inflammation, and renal fibrosis in remnant kidney through the Nrf2-keap1 pathway. Molecular Nutrition and Food Research 57 1649-1659. (doi:10.1002/mnfr.201200540)

Subramanian U, Kumar P, Mani I, Chen D, Kessler I, Periyasamy R, Raghavaraju G \& Pandey KN 2016 Retinoic acid and sodium butyrate suppress the cardiac expression of hypertrophic markers and proinflammatory mediators in Npr1 gene-disrupted haplotype mice. Physiological Genomics 48 477-490. (doi:10.1152/ physiolgenomics.00073.2015)

Vernia P, Monteleone G, Grandinetti G, Villotti G, Di Giulio E, Frieri G, Marcheggiano A, Pallone F, Caprilli R \& Torsoli A 2000 Combined oral sodium butyrate and mesalazine treatment compared to oral mesalazine alone in ulcerative colitis: randomized, double-blind, placebo-controlled pilot study. Digestive Diseases and Sciences $\mathbf{4 5}$ 976-981. (doi:10.1023/A:1005537411244)

Wu H, Zhou S, Kong L, Chen J, Feng W, Cai J, Miao L \& Tan Y 2014 Metallothionein deletion exacerbates intermittent hypoxia-induced renal injury in mice. Toxicology Letters 232 340-348. (doi:10.1016/j. toxlet.2014.11.015)

Wu H, Kong L, Cheng Y, Zhang Z, Wang Y, Luo M, Tan Y, Chen X, Miao L \& Cai L 2015a Metallothionein plays a prominent role in the prevention of diabetic nephropathy by sulforaphane via 
up-regulation of Nrf2. Free Radical Biology and Medicine 89 431-442. (doi:10.1016/j.freeradbiomed.2015.08.009)

Wu H, Kong L, Cheng Y, Zhang Z, Wang Y, Luo M, Tan Y, Chen X, Miao L \& Cai L 2015b Metallothionein plays a prominent role in the prevention of diabetic nephropathy by sulforaphane via up-regulation of Nrf2. Free Radical Biology and Medicine 89 431-442. (doi:10.1016/j.freeradbiomed.2015. 08.009)

Wu H, Kong L, Tan Y, Epstein PN, Zeng J, Gu J, Liang G, Kong M, Chen X, Miao L, et al. 2016 C66 ameliorates diabetic nephropathy in mice by both upregulating NRF2 function via increase in miR-200a and inhibiting miR-21. Diabetologia 59 1558-1568. (doi:10.1007/ s00125-016-3958-8)

Yaku K, Enami Y, Kurajyo C, Matsui-Yuasa I, Konishi Y \& Kojima-Yuasa A 2012 The enhancement of phase 2 enzyme activities by sodium butyrate in normal intestinal epithelial cells is associated with Nrf2 and p53. Molecular and Cellular Biochemistry 370 7-14. (doi:10.1007/ s11010-012-1392-x)
Yaku K, Matsui-Yuasa I, Konishi Y \& Kojima-Yuasa A 2013 AMPK synergizes with the combined treatment of 1 '-acetoxychavicol acetate and sodium butyrate to upregulate phase II detoxifying enzyme activities. Molecular Nutrition and Food Research 57 1198-1208. (doi:10.1002/mnfr.201200809)

Zhang DD \& Hannink M 2003 Distinct cysteine residues in Keap1 are required for Keap1-dependent ubiquitination of Nrf2 and for stabilization of Nrf2 by chemopreventive agents and oxidative stress. Molecular and Cellular Biology 23 8137-8151. (doi:10.1128/ MCB.23.22.8137-8151.2003)

Zhang MZ, Yao B, Yang S, Yang H, Wang S, Fan X, Yin H, Fogo AB, Moeckel GW \& Harris RC 2012 Intrarenal dopamine inhibits progression of diabetic nephropathy. Diabetes $612575-2584$. (doi:10.2337/db12-0046)

Zheng H, Whitman SA, Wu W, Wondrak GT, Wong PK, Fang D $\&$ Zhang DD 2011 Therapeutic potential of Nrf2 activators in streptozotocin-induced diabetic nephropathy. Diabetes 60 3055-3066. (doi:10.2337/db11-0807)

Received in final form 23 October 2016

Accepted 25 October 2016

Accepted Preprint published online 31 October 2016
Published by Bioscientifica Ltd 